\title{
Image-Based Feature Extraction Technique for Inclined Crack Quantification Using Pulsed Eddy Current
}

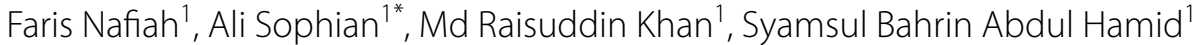 \\ and Ilham Mukriz Zainal Abidin²
}

\begin{abstract}
Existing eddy current non-destructive testing (NDT) techniques generally do not consider the inclination angle of inclined cracks, which potentially harms a larger region of a tested structure. This work proposes the use of 2D scan images generated by using pulsed eddy current (PEC) non-destructive testing (NDT) technique in the quantification of the inclination and depth of inclined cracks. The image-based feature extraction technique effectively identifies the crack axis, which consequently enables extraction of features from the extracted linear scans. The technique extracts linear scans from the images to allow the extraction of three novel image-based features, namely the length of extracted linear scans (LLS), the linear scan skewness (LSS), and the highest value on linear scan ( $\left(S_{\max }\right)$. The correlation of the three features to surface crack inclination angles and depths were analysed and found to be highly dependent on the crack depths, while only LLS and LSS are correlated to the crack inclination angles.
\end{abstract}

Keywords: Pulsed eddy current, 2D scan imaging, Feature extraction, Image processing, Inclined cracks

\section{Introduction}

As compared to conventional eddy current testing which only applies a single frequency for excitation, pulsed eddy current (PEC) offers the advantage of exciting magnetic field of multiple frequency components in a short period of time. PEC also offers many benefits over the more traditional eddy current NDT techniques, which makes it applicable in various applications as proposed by researchers around the globe. Additionally, PEC imaging techniques potentially serve as an effective tool to provide a more comprehensive understanding of the tested region, by constituting every pixel in $2 \mathrm{D}$ scan images with the peak value or other signal features obtained from differential signals at each spatial location. An extensive list of reported works suggested various approaches on crack quantifications and reconstruction through 2D scan technique. For example, He et al. [1] suggested the

\footnotetext{
*Correspondence: ali_sophian@iium.edu.my

1 Department of Mechatronics Engineering, Faculty of Engineering,

International Islamic University Malaysia, 53100 Kuala Lumpur, Malaysia Full list of author information is available at the end of the article
}

use of rectangular excitation coil to provide a constant distribution of magnetic field into the sample. Although the amount of magnetic field concentrated into the sample was not optimal, the results demonstrated a better predictive capability of identifying the width and length of the crack. In another work, Arjun et al. [2] studied the use of specially designed probe with a ferrite core in between the excitation and pick up coil to improve the performance of A-scan signals, thus allowing greater accuracy in 2D scan images. Besides defect characterisation, 2D scan images can also be employed for a fast crack profile reconstruction, as studied by Bai et al. [3]. PEC imaging technique was also proven to offer better reliability and defect detectability than flash thermography for crack detection in carbon fibre reinforced plastics (CFRP) [4].

Apart from probe scanning technique, Abrantes et al. [5], in his work, suggested the use of arrays of sensors in order to cover a larger area of inspection. Unlike a conventional excitation coil, a driver trace was placed between the $4 \times 4$ arrays of pick up coils, for the purpose of current excitation. The work in PEC was also extended 
to the use of measurement of the gradient of the magnetic flux density using gradient-field PEC (GPEC) probe, which was claimed to offer higher sensitivity $[6,7]$.

To the knowledge of the author, none of the literature explores the use of image-processing technique to extract linear scan responses from 2D scan images. Most of the time, the defects are detected by visually observing discontinuities in the acquired 2D scan images $[1,4,8,9]$. While, in some cases, features are extracted by manually determining a boundary point within the image instead of proposing an autonomous processing $[10,11]$. Literature also suggests a paper on using Fisher's linear discriminant analysis on PEC differential signals to classify defects of normal and inclined cracks but did not imply any size quantification [12]. Typical NDT techniques used for this purpose are PEC thermography [13] and ultrasonic testing (UT) [14-16].

Many simulations and validated experiments were designed to study the effects of crack depths to their corresponding PEC responses using either signals or images; however, little attention has been paid to find the theoretical influence of inclination angles. This is highly important in real life application, as cracks, such as rolling contact fatigue (RCF) in rail track heads, tend to grow at steep angles and can potentially result in an underestimation of the cracks. Since it is highly difficult to manufacture a calibration sample with specific crack geometry, well-defined cracks with different inclination angles are commonly used instead [13]. This research opens up the use of image processing to extract linear scans from 2D scan images. Features extracted from the obtained linear scan were compared with their corresponding inclination angles and depths. The rest of the paper is organized as follows: Section 2 discusses the methodology employed; Section 3 explains the results of the image-processing technique used, and the correlation of extracted features with crack parameters; Sections 4 presents the conclusions of the work.

\section{Experiment and Image Processing}

\subsection{PEC System Building}

A PEC system consisting of a DAQ system, an excitation circuit, and $x-y$ scanner and a probe was built. The general overview of the whole system is shown in Figure 1.

Aside from acquiring the excitation current and the response signals, the NI USB-6363 DAQ card serves as a scanner controller as well. At each scanning resolution, five pulses of amplitude of 9 A excitation current were supplied, with a width of $5 \mathrm{~ms}$, at $2.5 \mathrm{~Hz}$. The sampling rate was set to $100 \mathrm{ks} / \mathrm{s}$, acquired at each rising edge, with 30 pre-triggering samples being used to find the zero-Gauss voltage from the sensor output. The high

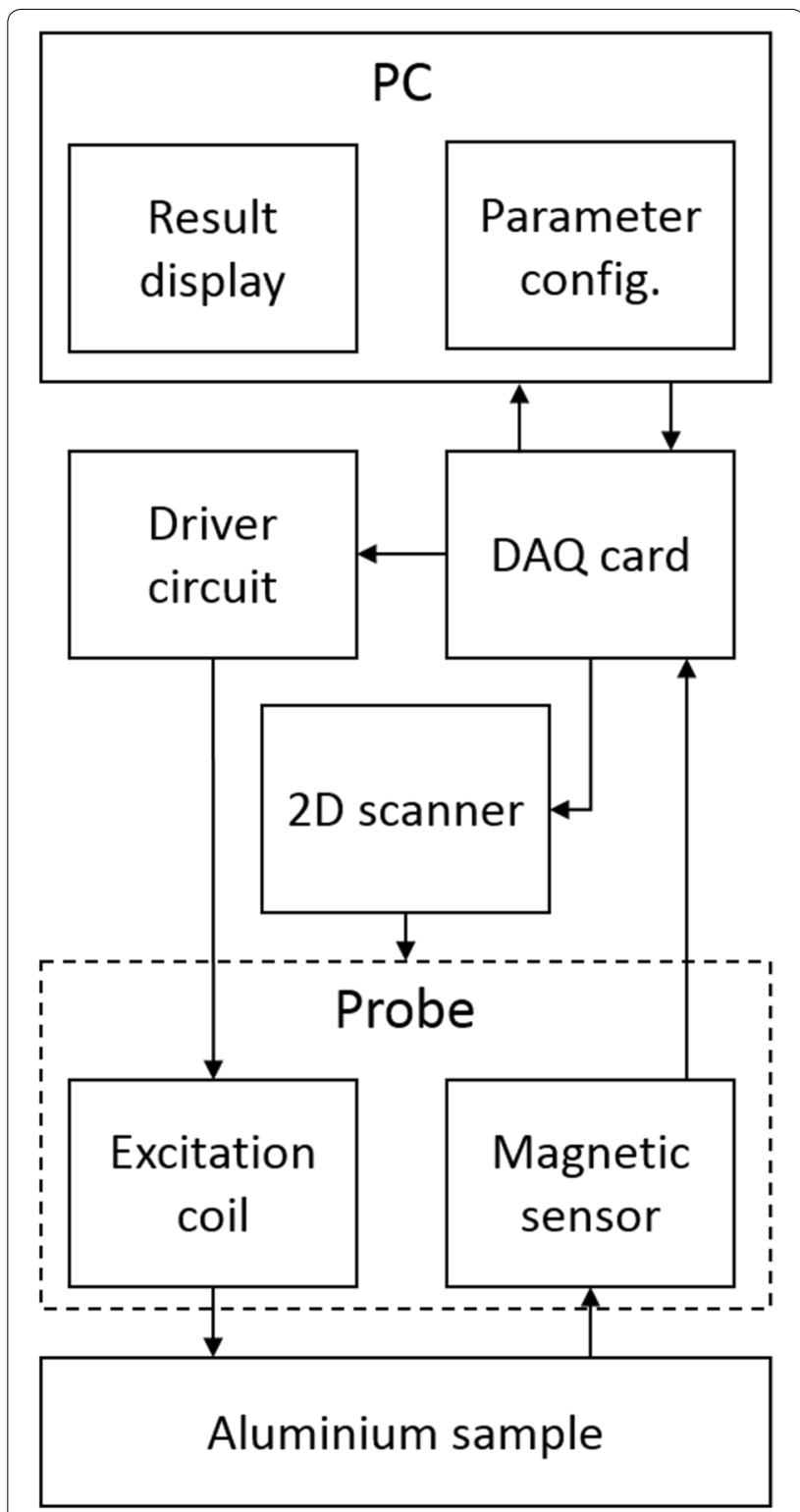

Figure 1 Block diagram of the developed PEC system

excitation current was supplied using a DC power supply, and to configure it to be a pulse, a Darlington transistor in a switch configuration was used [17]. The coil former was fabricated by using a 3D printer. The excitation coil has the following dimensions: $3.13 \mathrm{~mm}$ height, $6.5 \mathrm{~mm}$ inner radius and $10.04 \mathrm{~mm}$ outer radius, and 81 number of turns. A SS495A1 Hall sensor was placed in the middle of the coil. The design was studied using finite element modelling to find the optimal probe parameters to optimize the magnetic energy supply into the sample as well as to reduce the inductive effects on the time constant of the excitation current pulse. 


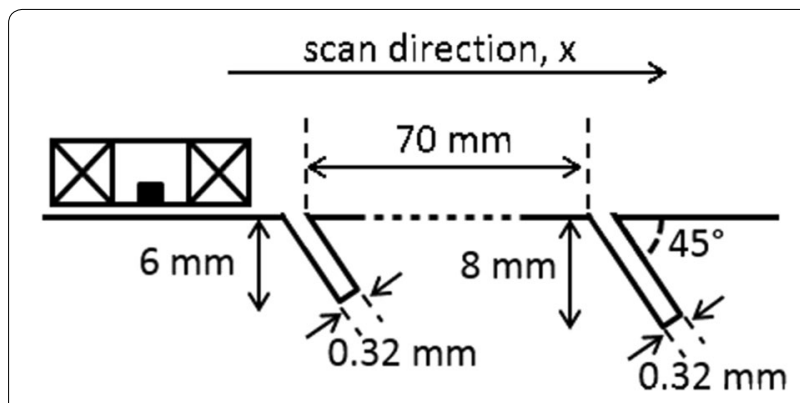

Figure 2 Simulated cracks of $45^{\circ}$ inclination angle, with $6 \mathrm{~mm}$ and 8 $\mathrm{mm}$ depths

Table 1 Crack parameters of different angles and depths for different data set

\begin{tabular}{lll}
\hline Crack angle $\left(^{\circ}\right)$ & Crack depth $(\mathbf{m m})$ & Width $(\mathbf{m m})$ \\
\hline $30 \pm 0.5$ & $(2,4,6,8) \pm 0.2$ & $0.35 \pm 0.05$ \\
$45 \pm 0.5$ & $(2,4,6,8) \pm 0.2$ & $0.35 \pm 0.05$ \\
$60 \pm 0.5$ & $(2,4,6,8) \pm 0.2$ & $0.35 \pm 0.05$ \\
$75 \pm 0.5$ & $(2,4,6,8) \pm 0.2$ & $0.35 \pm 0.05$ \\
$90 \pm 0.5$ & $(2,4,6,8) \pm 0.2$ & $0.35 \pm 0.05$ \\
\hline
\end{tabular}

\subsection{Data Collection}

To create the artificial cracks, slots were cut through the width of the aluminium test pieces, with different inclination angles and depths. Cracks were fabricated using electrical discharge machining (EDM) and separated by $70 \mathrm{~mm}$ from each other to avoid the edge effect. Figure 2 illustrates the fabricated cracks.

2D scan images for the cracks were constructed using differential signals obtained at each spatial resolution using scanning technique. Scanning was done around the simulated crack, to cover a square area of $30 \mathrm{~mm} \times 30 \mathrm{~mm}$, where the crack was approximately in the middle of the square. The PEC probe was then moved at an interval of $0.5 \mathrm{~mm}$ for the scanning purpose. Table 1 shows the parameters of the cracks.

The scanning was purposely done perpendicularly to the crack axis to collect more data from the 2D scan images, thus allowing greater number of data to be analysed. However, to demonstrate the effectiveness of the proposed image-based feature extraction technique, additional scanning was done on $45^{\circ}$ crack of $4 \mathrm{~mm}$ depth, in a random scanning direction.

The acquired response signals were filtered using low-pass filter and then normalized. The normalized reference signal from a flawless sample was subtracted from them to yield differential signals. Peak value was the feature used and plotted at each $2 \mathrm{D}$ coordinate to produce the $2 \mathrm{D}$ scan images. The $2 \mathrm{D}$ scan images were
2D-interpolated to improve the image resolution. For positioning reference, the signal with the highest peak value on one of the $2 \mathrm{D}$ scan images row was assigned as the $0-\mathrm{mm}$ position. Bearing in mind that the surface crack grows to the right-hand side of the crack opening, the signals that are picked up before reaching the differential signal with the highest peak were assigned as the signals at negative positions.

\subsection{Image-Based Feature Extraction Technique}

As the crack extended completely across the width of the test piece, the highest peak values in the $2 \mathrm{D}$ scan images contributed to a line across the image, which should be along the direction of the crack axis. To make full use of it, the pixels in each image containing the highest peak values (local maxima) were first detected. It is also important to note that finding local maxima in an image can be troublesome when the image is noisy. Noise typically contributes to single pixel variation, thus making the algorithm to find local maxima to be ineffective. Prior to the steps, median filtering was first applied.

The proposed technique for processing 2D scan images composed of four main routines; image dilation, nonmaximum suppression, the Hough transform, and the extraction of pixel value orthogonal to the crack axis, which are discussed below.

\subsubsection{Image Dilation}

Image dilation used structuring element (can be of various shapes and sizes) to be probed at all possible positions throughout the $2 \mathrm{D}$ scan images. The image pixel at the centre of the structuring element was compared to the neighbouring pixel values within the structuring element, and the highest pixel value was then assigned as the corresponding pixel value in the output image. The output image of an image dilation contained an enlarged region of local maxima. In this work, diamond-shaped structuring element of a radius of 1 pixel was employed.

\subsubsection{Non-maximum Suppression}

Each pixel in the input image was then compared to the corresponding pixel in the dilated image, by taking the absolute value of the difference between the input image and the dilated image pixel values. The value of ' 1 ' is assigned in a new binary image if these conditions were met: (1) The absolute value obtained was lower than a threshold of '0', and (2) the input image pixel was checked if having the value of lower than 0.01 , to remove false interpretation of local maxima. When the mentioned conditions were not met, the new binary image pixel was assigned as ' 0 '. The outputs of this routine were binary images of detected local maxima and non-local maxima within the image. 


\subsubsection{Hough Transform}

Hough transform attempts to construct lines through the data lines and data points present in the binary image, in all possible directions. The constructed lines passing through each other were therefore grouped together. The data lines and data points where the constructed lines originated from were assigned as one single line. For each data points (or lines) in the spatial domain, a number of lines were plotted going through it, all at different angles. The distance between the line to the origin was also computed. Both angle and distance information were then transferred into the polar domain. This was repeated for all data points. Data points sharing the same value of angle and distance were grouped together and treated as a line. Grouping of the angle and distance values are also termed as 'bin'. To make the algorithm robust to the noises present in the image, the bin tolerance can be adjusted. Hough transform provided the information regarding the length and angle of the lines, which can be transferred back to the 2D scan images for better isolation of cracks.

\subsubsection{Extracting Pixel Values Orthogonal to Crack Axis}

Extracting pixel values starting from the detected crack line to the boundary of the 2D scan image requires a massive amount of memory in real life inspection. It can also provide false interpretation of the data. Absolute threshold was not an option as it imposed a larger region of inspection. Instead, a threshold of $60 \%$ from the local maxima on each row was made as the linear scan limit. While extracting pixel values from both positive and negative positions with respect to each row's local maxima, the pixel value was monitored if it has reached the $60 \%$ threshold. Empirically, a relative threshold of $60 \%$ of the local maxima only required a length of less than $7 \mathrm{~mm}$ on both positive and negative sides of the local maxima. Although it is arguable that $60 \%$ was chosen rather at random, it served the intention of extracting a linear scan of acceptable length as well as containing reliable features for crack parameter quantification.

The crack axis lines detected were first extrapolated to the edge of the images as the crack was recognized to extend across the 2D scan images. Figures 3(a) and (b) depict the illustration of extracting linear scan from $45^{\circ}$, $4 \mathrm{~mm}$ crack 2D scan image on one of the points along the crack axis line. From that, the pixel values orthogonal to each point along the detected line were extracted. Using simple geometry, crack direction, $\Phi$, obtained from Hough transform was used to compute the coordinate of $x 1, x 2, y 1$, and $y 2$ as shown in Eq. (1):

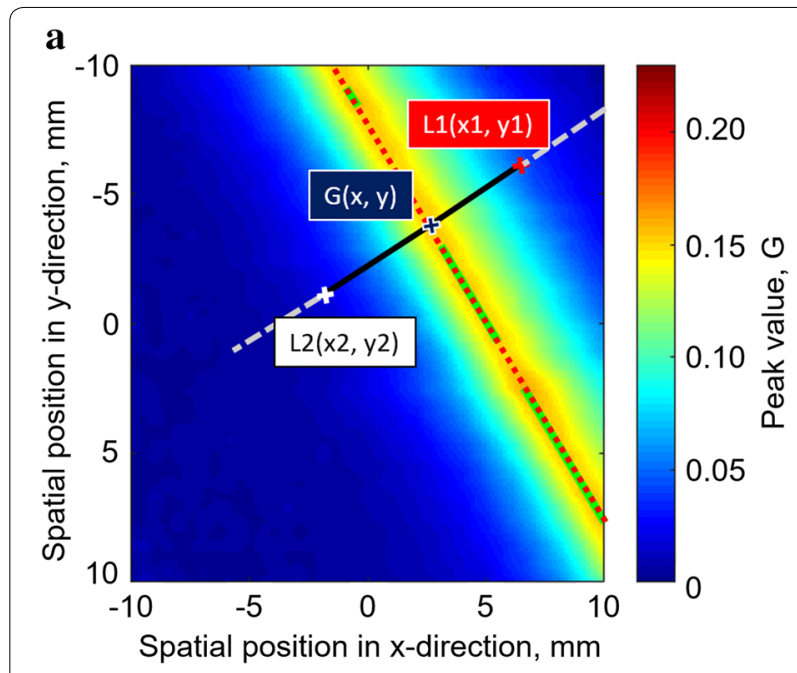

b

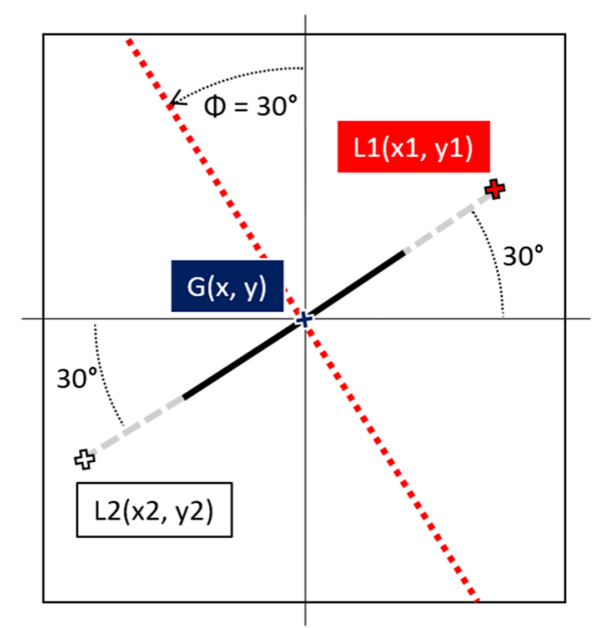

Figure 3 (a) Linear scan orthogonal to the extrapolated crack axis, and (b) illustration of the extraction of linear scan in a vector space

$$
\begin{aligned}
& x 1=\Phi+\text { length } \times \cos (\Phi), \\
& x 2=-\Phi+\text { length } \times \cos (-\Phi), \\
& y 1=\Phi+\text { length } \times \cos (\Phi), \\
& y 2=-\Phi+\text { length } \times \cos (-\Phi) .
\end{aligned}
$$

The value of length was arbitrarily chosen, as long as it permitted the $60 \%$ threshold of the linear scan, as the remaining pixel values after thresholding will be discarded.

The summary of the whole strategy for feature extraction is shown in Algorithm 1. 


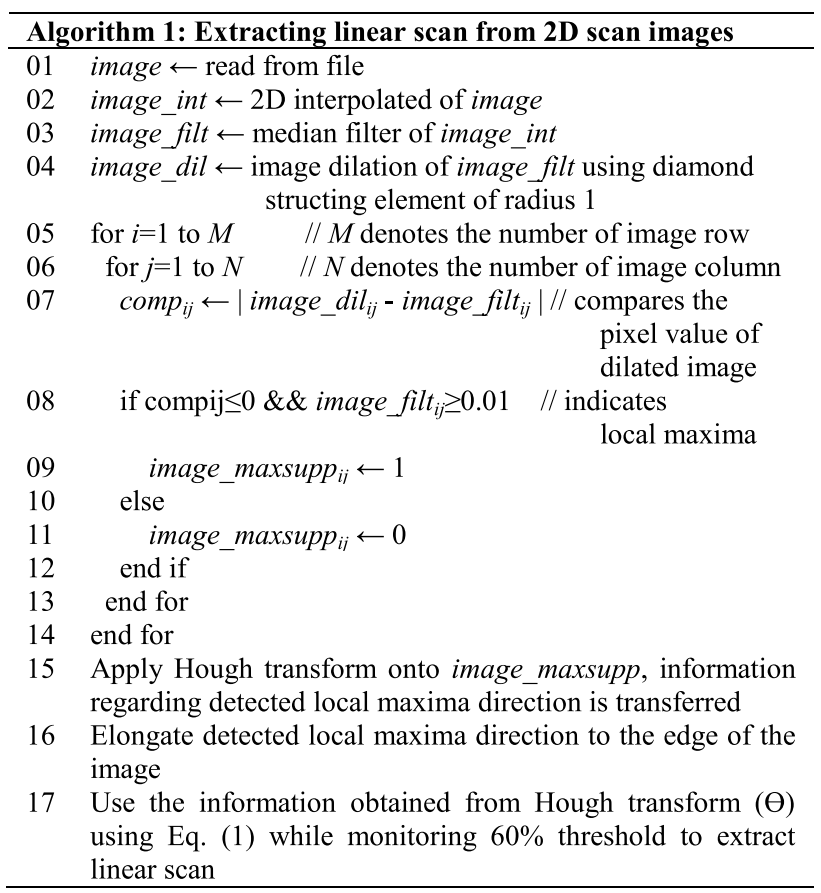

\subsection{Novel Features from 2D Scan Image}

Three novel image-based features were used to effectively quantify surface crack inclination angles and depths, namely length of linear scan (LLS), linear scan skewness (LSS), and linear scan peak ( $\left(\mathrm{S}_{\max }\right)$. The LLS is essentially the length of the linear scan, after $60 \%$ thresholding. The feature was opted as eddy current tend to concentrate the eddy current along the crack elongation, making the LLS to increase with the decrease of inclination angle.

The symmetricity of the 2D scan images around the crack axis was observed to differ across distinct inclination angles. Hence, the second image-based feature was introduced, termed as LSS. LSS explains the effect of the crack inclination angles on the field distribution at different probe positions. The elongation of inclined cracks to the positive spatial positions made the linear scan to settle slower than the negative side. The calculation of LSS was adopted from statistical skewness function [18], which can be defined as

$$
L S S=\frac{\sum_{i=1}^{N}\left[\left(x_{i}-\bar{x}\right)^{3} \times p_{i}\right]}{\left\{\sum_{i=1}^{N}\left[\left(x_{i}-\bar{x}\right)^{2} \times p_{i}\right]^{2}\right\}^{\frac{3}{2}}},
$$

where $p_{i}$ was the $i$ th peak value, $x_{i}$ was the $i$ th spatial position, $N$ was the total number of spatial positions. Similar to the formula of mean in statistics, $\bar{x}$ was the first moment of the distribution function, calculated as

$$
\bar{x}=\frac{\sum_{i=1}^{N}\left(x_{i} \times p_{i}\right)}{\sum_{i=1}^{N} p_{i}} .
$$

The third feature was termed as $\mathrm{LS}_{\max }$, coming from the extraction of maximum value of each linear scan. According to our previous work, the maximum peak value of LLS does not occur exactly above the middle of crack opening, but slightly further to the crack elongation direction [19].

\section{Results and Discussions}

The first section of the results will discuss the effectiveness of the image processing technique employed, while the second will discuss the analysis on the proposed features.

\subsection{Reliability of the Image-Based Feature Extraction Technique}

The reliability of the proposed technique was proven with the detected line segments on the tested 2D scan image, as depicted in Figure 4(a) and (b). As seen in Figure 4(a), the non-maximum pixel value was effectively suppressed, allowing only maximum pixel value to be present in the image. Although not shown in this paper, signals with low magnitude demonstrated noises, which can be falsely interpreted as local maxima. However, the voting procedure enclosed in Hough transform favourably detected the densest region in the Hough matrices to be treated as a line. Although the results of employing the proposed feature extraction technique were effective for surface cracks, it was observed that the technique can still be improved as it was not robust towards noise, as empirically tested on 2D scan images of subsurface cracks. Shallow cracks contained relatively low peak values, thus resulting in lower signal-to-noise ratio. "Salt and pepper" noise in image creates a 1-pixel variation, which typically caused the algorithm to falsely detect the pixel as a peak.

\subsection{Analysis of the Proposed Features 3.2.1 Feature Correlation Analysis}

The coefficient correlation matrix, $\mathrm{R}$, for all features collected from each 2D scan image, was constructed to analyse feature redundancy, as shown in Table 2. Looking at the off-diagonal matrix values, the three features from $2 \mathrm{D}$ scan images constructed using peak value were proven to be uncorrelated, as convinced by the $\mathrm{R}$ value lower than 0.8 . Thus, they did not provide redundant information in building a regression model. 

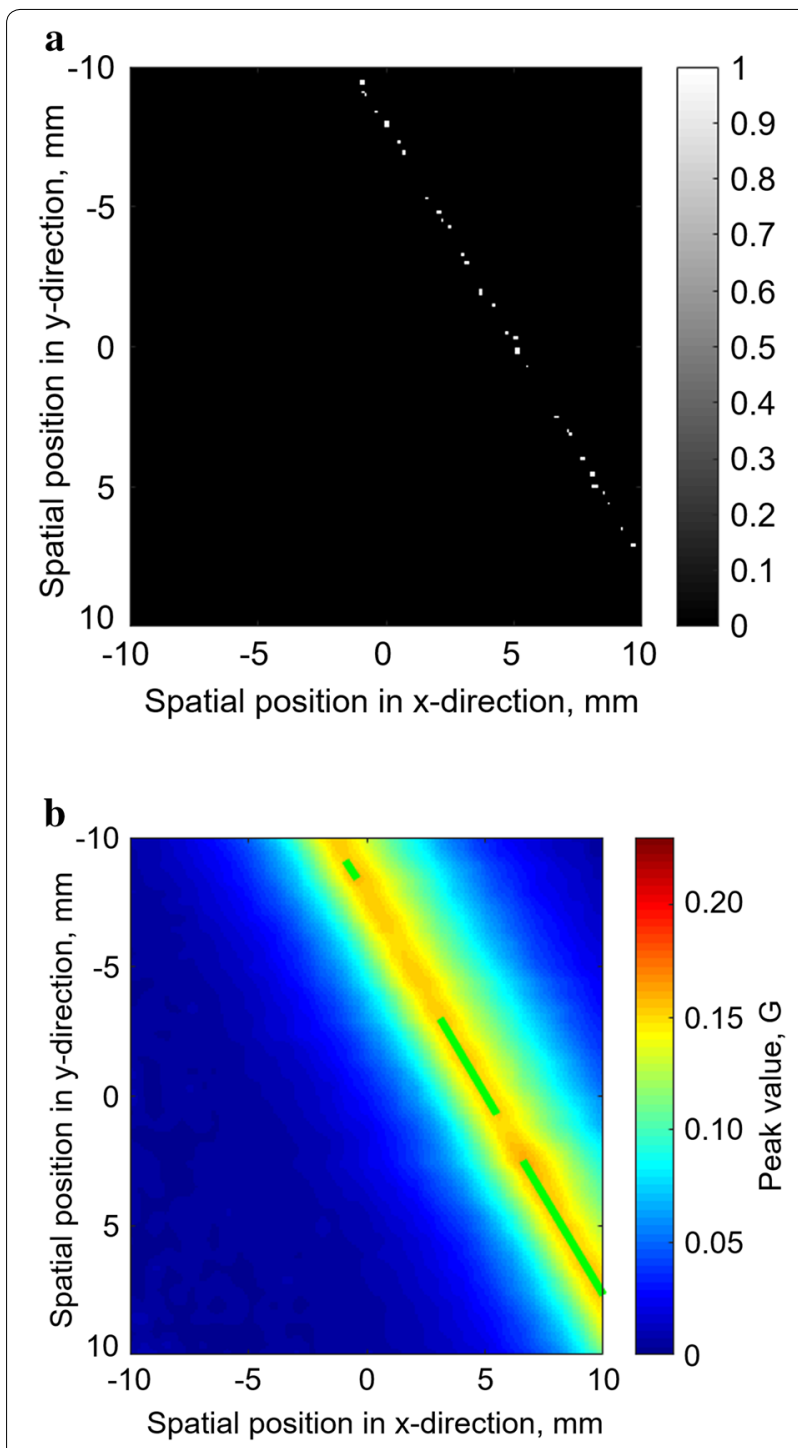

Figure 4 Proposed image-based feature extraction technique on additional scanning of crack of $45^{\circ}, 4 \mathrm{~mm}$, where (a) is the binary image containing crack direction and $(\mathbf{b})$ is the detected line

Table 2 Correlation matrix of all features

\begin{tabular}{llll}
\hline & LLS & LSS & LS $_{\max }$ \\
\hline LLS & 1 & 0.7966 & 0.4636 \\
LSS & 0.7966 & 1 & 0.2946 \\
LS $_{\max }$ & 0.4636 & 0.2946 & 1 \\
\hline
\end{tabular}

\subsubsection{Feature Analysis}

Figure 5(a) shows the relation between the LLS with inclination angles at different depths and Figure 5(b) shows the feature's relation with depth at different inclination angles. From both figures, it can be concluded that both crack parameters influenced the trend of the LLS. As depicted in Figure 5(a), the correlation between the LLS on the inclination angle can be claimed to be quadratic of different coefficients at different depths. As the inclination angle decreased, the crack tended to extend further. This contributed to the formation of eddy currents along the crack growth, while having relatively more effect on the response signals. The LLS generally increased with the increase of depth. In terms of PEC scanning, deeper cracks responded more to the divergence of the supplied field, which consequently gave higher amplitudes of differential signals at positions further from the crack opening. Figure 5(b) also shows a more significant influence of depth on the LLS with smaller inclination angles, since the growth of the cracks made the crack tip to be further from the crack opening.

Figures 6(a) and (b) shows the relationship of the LSS and crack parameters. From both figures, the LSS was mostly linearly dependent on both inclination angles and depths. LSS explained the measure of symmetricity of the cracks, with $90^{\circ}$ cracks ideally exhibited zero LSS. While this was true, crack depths also influenced LSS because of the high concentration of eddy current for cracks of deeper depths. Inclined deeper cracks also lengthen further, resulting in higher LSS. It was also observed that despite the depths, the LSS for cracks of $90^{\circ}$ was almost flat at zero value, explaining the symmetrical effect of magnetic field at both side of the linear scanning. The LSS was also more prone to noise as observed at the standard deviations, since the calculation of the LSS itself considered the peak value at each spatial position.

Interestingly, $\mathrm{LS}_{\max }$ correlates strongly with depth, but inclination angle has relatively less effect on $\mathrm{LS}_{\max }$, as illustrated in Figures 7(a) and (b). Although in theory, inclination angle wass believed to provide slight influence on $\mathrm{LS}_{\max }$ due to the crack elongation, but practical application proved otherwise due to the limited effect of the inclination angle on the response signals.

\section{Conclusions}

Inclined cracks have been proven to have different influences on the peak value of the differential signal at different spatial positions. Three features have been proposed previously, namely LLS, LSS and $\mathrm{LS}_{\max }$. In order to extract those features, an image-based feature extraction technique has been proposed, which was empirically observed to be effective in exploiting surface 

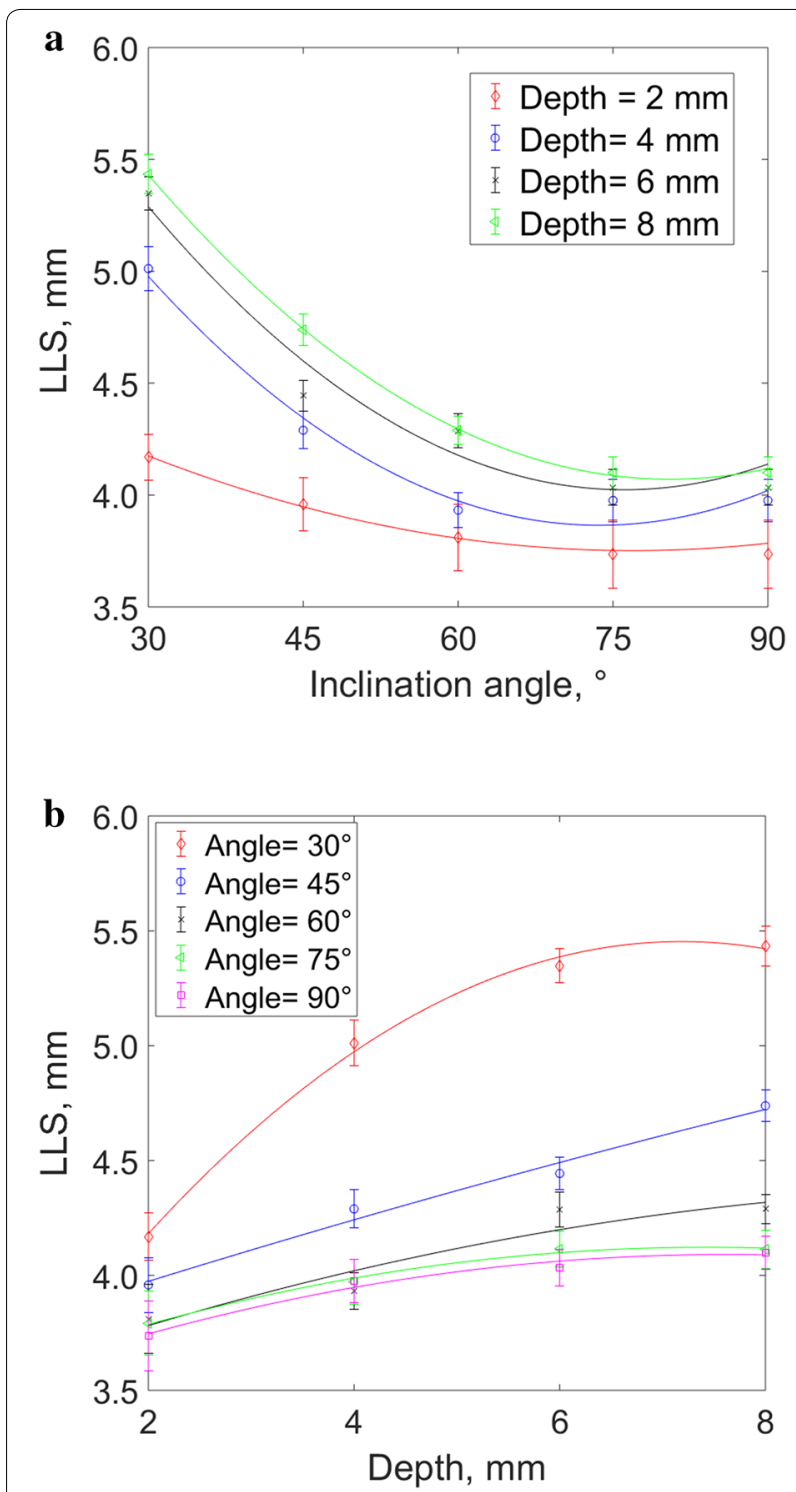

Figure 5 Correlation of LLS with (a) inclination angle, at different depths and (b) depth, at different inclination angle
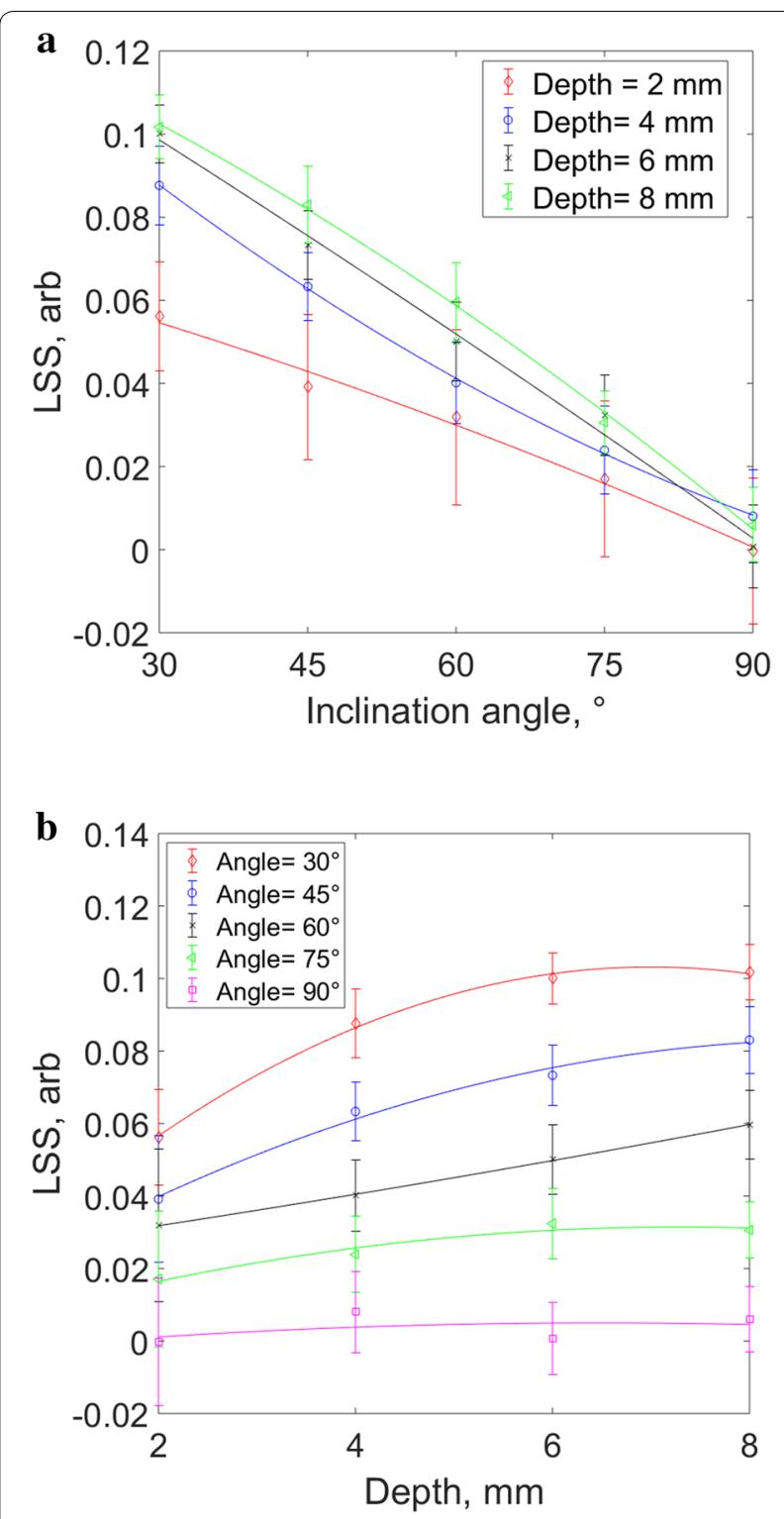

Figure 6 Correlation of LSS with (a) inclination angle, at different depths and (b) depth, at different inclination angle crack's 2D scan images for automatic crack direction detection.

Experimental measurements demonstrated a basis for developing an inverse model for defect quantification.
The $\mathrm{LS}_{\max }$ was proven to be less affected by the inclination angle, which served as a good feature for depth approximation. Meanwhile, the linear correlation of the LLS and the LSS is useful for inclination angle 

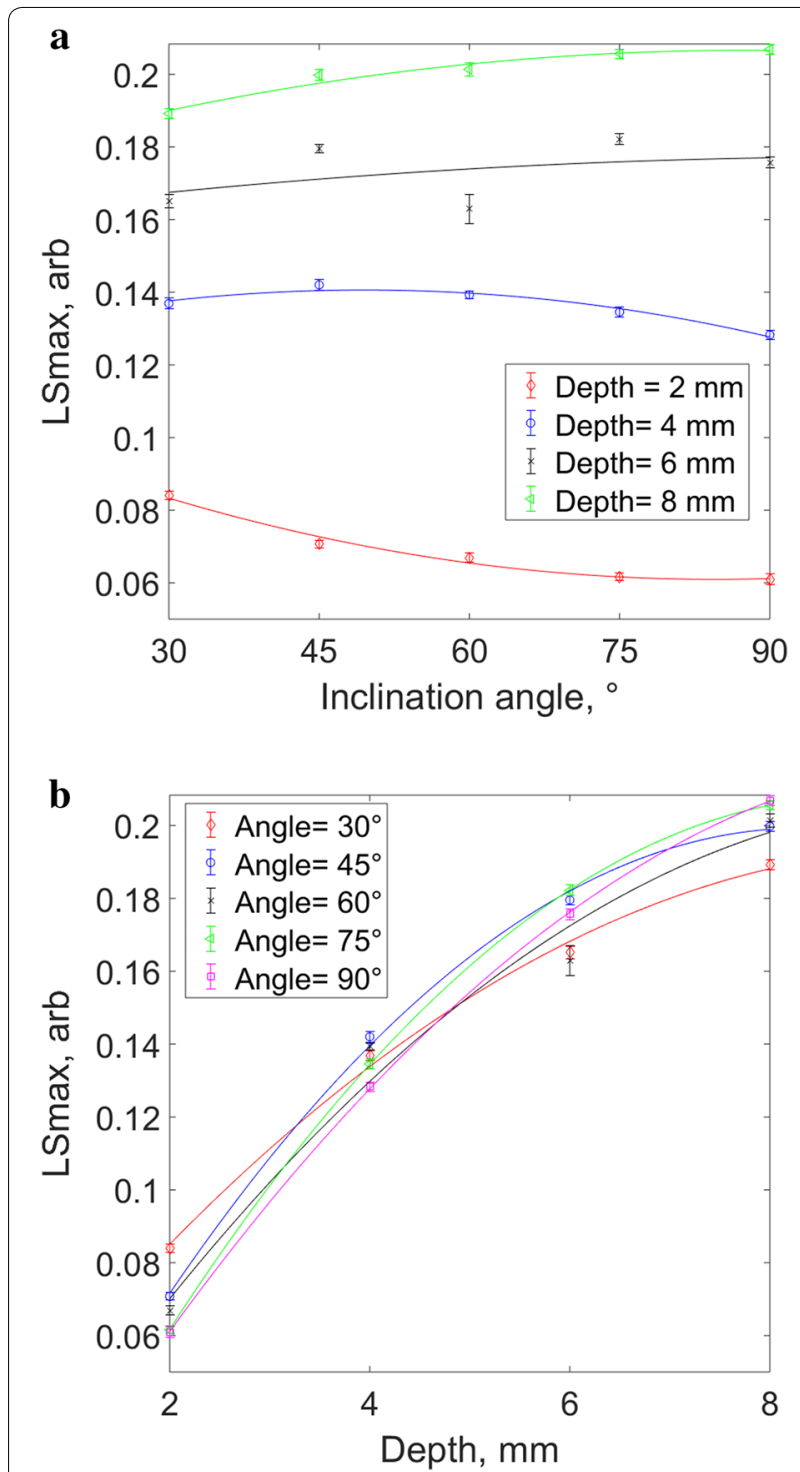

Figure 7 Correlation of $\mathrm{LS}_{\max }$ with (a) inclination angle, at different depths and (b) depth, at different inclination angle

quantification, with the prior knowledge of the depth. The extension of the simultaneous characterisation of both inclination angles and depths are reserved for future works.

\section{Authors' Contributions}

FN and AS conceived the presented idea. FN carried out the experiment and analysed the presented results. FN and AS wrote the manuscript with support from MRK, SBAH, and IMZA. All authors read and approved the final manuscript.

\section{Author Details}

${ }^{1}$ Department of Mechatronics Engineering, Faculty of Engineering, International Islamic University Malaysia, 53100 Kuala Lumpur, Malaysia. ${ }^{2}$ Leading Edge NDT Group, Malaysian Nuclear Agency, 43000 Bangi, Selangor, Malaysia.

\section{Authors' Information}

Faris Nafiah, born in 1993, is currently a PhD candidate at London South Bank University, U.K. He received his BEng. (Hons.) from the University of Auckland, New Zealand in 2015, and his Msc in Mechatronics Engineering from International Islamic University Malaysia, Malaysia in 2018. His research interest includes eddy current NDT and signal processing.

Ali Sophian is currently an assistant professor at International Islamic University Malaysia. He received his PhD from the University of Huddersfield, U.K., in 2004. His research interests include eddy current NDT and signal processing.

Md Raisuddin Khan received this PhD in Mechanical Engineering from Bangladesh University of Engineering and Technology (BUET). He is currently a professor at Department of Mechatronics Engineering, International Islamic University Malaysia. His research area is robotics, vibration and mechanism synthesis.

Ilham Mukriz Zainal Abidin, a research officer at the Leading Edge NonDestructive Testing Technology (LENDT) Group, Malaysian Nuclear Agency, received his PhD in 2010 from Newcastle University, U.K. His research area includes active thermography and advanced NDT.

Syamsul Bahrin A. Hamid, an assistant professor at International Islamic University Malaysia, received his PhD from the University of Strathclyde. His research interest is in electromechanical power engineering.

\section{Competing Interests}

The authors declare that they have no competing interests.

\section{Funding}

Supported by Malaysia's Ministry of Higher Education (Grant No.

FRGS16-059-0558).

Received: 21 March 2018 Accepted: 11 March 2019

Published online: 25 March 2019

\section{References}

[1] Y He, M Pan, F Luo, et al. Pulsed eddy current imaging and frequency spectrum analysis for hidden defect nondestructive testing and evaluation. NDT and E International, 2011, 44(4): 344-352. https://doi. org/10.1016/j.ndteint.2011.01.009.

[2] V Arjun, B Sasi, B P C Rao, et al. Optimisation of pulsed eddy current probe for detection of sub-surface defects in stainless steel plates. Sensors and Actuators A: Physical, 2015, 226: 69-75. https://doi.org/10.1016/j. sna.2015.02.018.

[3] L Bai, G Yun Tian, A Simm, et al. Fast crack profile reconstruction using pulsed eddy current signals. NDT \& E International, 2013, 54: 37-44. https://doi.org/10.1016/j.ndteint.2012.11.003.

[4] Y He, G Tian, M Pan, et al. Non-destructive testing of low-energy impact in CFRP laminates and interior defects in honeycomb sandwich using scanning pulsed eddy current. Composites Part B: Engineering, 2014, 59: 196-203. http://dx.doi.org/10.1016/j.compositesb.2013.12.005.

[5] R F Abrantes, L S Rosado, M Piedade, et al. Pulsed eddy currents testing using a planar matrix probe. Measurement: Journal of the International Measurement Confederation, 2016, 77: 351-361. https://doi.org/10.1016/j. measurement.2015.09.026.

[6] Y Li, H Jing, I Mukriz, et al. A gradient-field pulsed eddy current probe for evaluation of hidden material degradation in. Sensors, 2017, 17(943): https://doi.org/10.3390/s17050943.

[7] $Y$ Li, H Jing, I Mukriz, et al. Gradient-field pulsed eddy current probes for imaging of hidden corrosion in conductive structures. Sensors and Actuators A: Physical, 2016, 238: 251-265. https://doi.org/10.1016/j.sna.2015.12.026.

[8] M Pan, Y He, G Tian, et al. Defect characterisation using pulsed eddy current thermography under transmission mode and NDT applications. NDT and E International, 2012, 52: 28-36. https://doi.org/10.1016/j.ndtei nt.2012.08.007.

[9] Y He, F Luo, M Pan. Defect characterisation based on pulsed eddy current imaging technique. Sensors and Actuators, A: Physical, 2010, 164(1-2): 1-7. https://doi.org/10.1016/j.sna.2010.09.001.

[10] J Kim, G Yang, L Udpa, et al. Classification of pulsed eddy current GMR data on aircraft structures. NDT \& E International, 2010, 43(2): 141-144. https://doi.org/10.1016/j.ndteint.2009.10.003. 
[11] G Yang, A Tamburrino, L Udpa, et al. Pulsed eddy-current based giant magnetoresistive system for the inspection of aircraft structures. IEEE Transactions on Magnetics, 2010, 46(3): 910-917. https://doi.org/10.1109/ tmag.2009.2032330.

[12] X Chen, D Hou, L Zhao, et al. Study on defect classification in multi-layer structures based on Fisher linear discriminate analysis by using pulsed eddy current technique. NDT and E International, 2014, 67: 46-54. https:// doi.org/10.1016/j.ndteint.2014.07.003.

[13] I Zainal Abidin, G Yun Tian, J Wilson, et al. Quantitative evaluation of angular defects by pulsed eddy current thermography. NDT and E International, 2010, 43(7): 537-546. https://doi.org/10.1016/j.ndteint.2010.05.010.

[14] B Dutton, A R Clough, M H Rosli, et al. Non-contact ultrasonic detection of angled surface defects. NDT and E International, 2011, 44(4): 353-360. https://doi.org/10.1016/j.ndteint.2011.02.001.
[15] P A Petcher, M D G Potter, S Dixon. A new electromagnetic acoustic transducer (EMAT) design for operation on rail. NDT and E International, 2014 65: 1-7. https://doi.org/10.1016/j.ndteint.2014.03.007.

[16] C B Thring, Y Fan, R S Edwards. Focused Rayleigh wave EMAT for characterisation of surface-breaking defects. NDT and E International, 2017, 88: 1-7. https://doi.org/10.1016/j.ndteint.2017.02.005.

[17[ P Horowitz, W Hill. The art of electronics. 3rd ed. New York: Cambridge University Press, 2015.

[18] M S Srivastava. A measure of skewness and kurtosis and a graphical method for assessing multivariate normality. Statistics \& Probability Letters, 1984, 2: 263-267. https://doi.org/10.1016/0167-7152(84)90062-2.

[19] F Nafiah, A Sophian. Pulsed eddy current imaging of inclined surface cracks. Indonesian Journal of Electrical Engineering and Informatics, 2017 5(4): https://doi.org/10.11591/ijeei.v5i4.359.

\section{Submit your manuscript to a SpringerOpen ${ }^{\circ}$ journal and benefit from:}

- Convenient online submission

- Rigorous peer review

- Open access: articles freely available online

- High visibility within the field

- Retaining the copyright to your article

Submit your next manuscript at $\boldsymbol{\nabla}$ springeropen.com 